\title{
Modeling Current and Future Potential Geographical Distribution of Carpinus tientaiensis, a Critically Endangered Species from China
}

\author{
Runan Zhao ${ }^{1,2} \oplus$, Xiaojie Chu ${ }^{3}$, Qianqian He ${ }^{1,2}$, Yan Tang ${ }^{1,2}$, Min Song ${ }^{1,2}$ and Zunling Zhu ${ }^{1,2, *}$ \\ 1 College of Landscape Architecture, Nanjing Forestry University, Nanjing 210037, China; \\ zhao-rn@njfu.edu.cn (R.Z.); heqianqian@njfu.edu.cn (Q.H.); tangyan@njfu.edu.cn (Y.T.); \\ songmin@njfu.edu.cn (M.S.) \\ 2 Co-Innovation Center for Sustainable Forestry in Southern China, Nanjing Forestry University, \\ Nanjing 210037, China \\ 3 College of Chemistry and Life Sciences, Zhejiang Normal University, Jinhua 321004, China; \\ chu-xj@zjnu.edu.cn \\ * Correspondence: zhuzunling@njfu.edu.cn; Tel.: +86-025-69638089
}

Received: 10 June 2020; Accepted: 16 July 2020; Published: 19 July 2020

\begin{abstract}
Future climate change will have serious impacts on species survival and distribution and will likely lead to the extinction of some species classified as endangered. Carpinus tientaiensis (Betulaceae), a unique and endangered species in China, has restricted distribution and a small population, indicating an urgent need for its protection. However, research on its current distribution or the influence that climate change will have on its future survival and distribution is limited. We used a MaxEnt model and ArcGIS software to predict the current and future niches of $C$. tientaiensis. The current suitable distribution area of $C$. tientaiensis is small, mainly in east China, south Zhejiang and Anhui, and central and southern mountainous areas of Taiwan province. The core suitable areas are concentrated in the Xianxialing and Kuocang mountains in south Zhejiang, the southern mountains of Taiwan, and the Dabie, Huangshan and Jiuhua mountains in south Anhui. Among the 15 BIOCLIM variables examined, the precipitation of the driest quarter (bio17) was found to be the most important factor limiting $C$. tientaiensis survival and distribution. Future field investigations will focus on the Xianxialing and Kuocang mountains, as they may have unidentified wild C. tientaiensis communities. In the future, the Kuocang, Dapan and Tiantai mountains in east Zhejiang, and the high-altitude areas of Dabie and Jiuhua mountains in south Anhui, will be suitable for $C$. tientaiensis ex situ conservation and cultivation. However, the suitable distribution and core suitable areas for $C$. tientaiensis will decrease sharply as they are susceptible to climate shocks. Moreover, the suitable distribution area of C. tientaiensis is predicted to move slightly north and obviously eastward. Therefore, we suggest that strengthen conservation and management efforts for $C$. tientaiensis in its original habitats, and actively carry out ex situ conservation and artificial breeding in botanical gardens.
\end{abstract}

Keywords: Carpinus tientaiensis; endangered species; MaxEnt model; potential distribution; climate change; protective measures

\section{Introduction}

The geographical distribution of species, including plants, is closely related to the environmental conditions, and climatic factors play a decisive role in this [1]. In recent years, climate warming has increased because of climate change [2], and this has already had a serious impact on species distributions [3]. Research has shown that as climate change continues to increase in the future, species will gradually migrate to higher altitude or latitude areas [4], resulting in a decrease in species 
habitats [5]. Consequently, for endangered species, this may lead to extinction or the increased risk thereof [6,7]. In addition, habitat fragmentation can have adverse effects on the survival and distribution of a species, leading to the loss of biodiversity and species extinction [8,9]. Generally, endangered species have a small number of individuals; they also have special environmental needs, and their habitats often show obvious fragmentation. It is important to evaluate the influence of the climate and other environmental factors on the habitats and distributions of endangered plants with appropriate scientific methods, to help prevent the extinction of species and preserve biodiversity.

With the development of geographic information technologies, species distribution models (SDMs) have become an important technical means by which to predict species distribution ranges and potential distributions of species [10]. However, at present, there have only been a few studies on endangered trees [11], as data about the distribution of endangered plants are scarce and difficult to obtain [12,13]. The MaxEnt model [14] is a niche model based on the principle of maximum entropy; the model can achieve good results even when the data about the distribution of a species are scarce [13,15]. It has previously been widely used to predict the distributions of endangered species [16,17]. It also provides further reference information that can help to protect endangered species, such as predicting species distributions, assessing how they are constrained by environmental conditions, identifying the impact of environmental changes on their distributions, and determining potential areas for the reintroduction of rare species.

Carpinus tientaiensis Cheng is a deciduous tree from the Betulaceae family (Figure 1). It is a unique and endangered Tertiary relict plant species from China [18,19]. Currently, wild C. tientaiensis are only known to be distributed in parts of Zhejiang province-Tiantai mountain in Tiantai county, Dapan mountain in Pan'an county, Yangtianhe in Qingtian county, and Shangshantou in Jingning She Autonomous county. It is listed on the Red List of critically endangered species of the International Union for the Conservation of Nature (IUCN, http://www.iucnredlist.org) [20], as its wild populations are small and its habitat is currently decreasing, leaving the species facing a risk of extinction. C. tientaiensis also has a high level of scientific research value for the fields of plant geography and species diversity formation [21]. The habitat of C. tientaiensis is restricted and seriously threatened, and the species also has a weak ability to adapt to new environments; this means there is an urgent need for its current distribution to be understood and for its natural habitats to be preserved.

At present, it is not clear how climate change will affect the distribution of $C$. tientaiensis in the future. Based on field investigations, this study used a MaxEnt model to project the current and future distribution of $C$. tientaiensis. The aim was to further understand the distribution patterns and potential distribution areas of $C$. tientaiensis, to provide reference data to help locate unknown wild communities. The suitable distribution areas and core suitable areas of $C$. tientaiensis, as well as the main environmental factors limiting its survival and distribution were also identified. This information will facilitate the species introduction, conservation, and cultivation in the future. Based on the impacts that climate change will have on the suitable distribution areas for $C$. tientaiensis, corresponding protection strategies could reduce the impacts of climate change on the wild communities of $C$. tientaiensis.

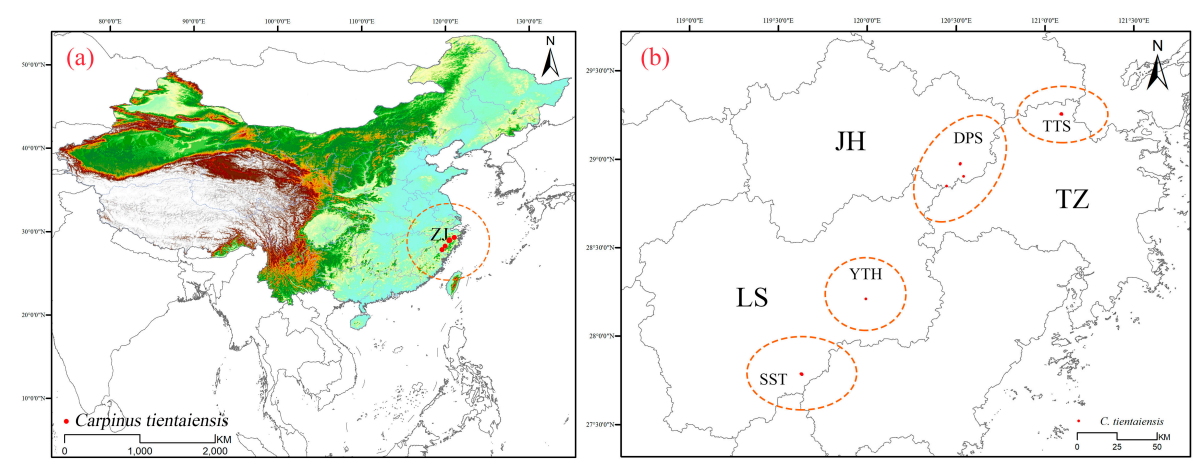

Figure 1. Cont. 

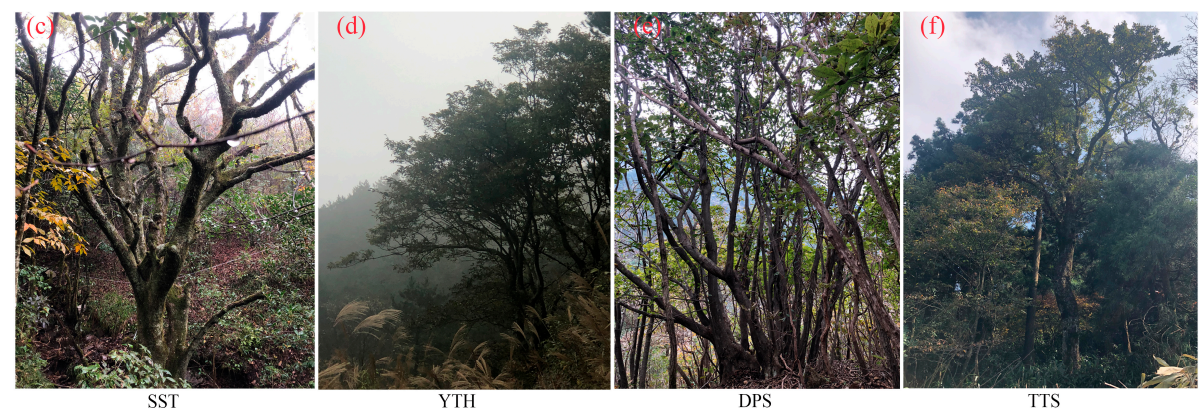

Figure 1. Current locations of Carpinus tientaiensis populations in China. (a) Geographic locations of C. tientaiensis populations in China; (b) the geographical location of $C$. tientaiensis in Zhejiang Province; (c-f) field photos of C. tientaiensis located in Shangshantou (SST) in Jingning She Autonomous county, Yangtianhe (YTH) in Qingtian county, Dapan mountain (DPS) in Pan'an county and Tiantai mountain (TTS) in Tiantai county, respectively. ZJ: Zhejiang province; JH: Jinhua city; TZ: Taizhou city; LS: Lishui city. (The field pictures of $C$. tientaiensis were taken by R.Z.).

\section{Materials and Methods}

\subsection{Species Distribution Data}

C. tientaiensis is an endemic and endangered plant in China and its only known distribution area is in Zhejiang province. On the basis of consulting the Flora of China [19] and the Chinese Virtual Herbarium (http:v5.cvh.ac.cn/), a detailed investigation of the distribution of C. tientaiensis was carried out in Zhejiang province from 2018 to 2019, and the longitude and latitude of each C. tientaiensis were recorded. This resulted in the distribution data for 58 plants (Table S1) across the following four regions: Tiantai mountain in Tiantai county, Dapan mountain in Pan'an county, Yangtianhe in Qingtian county, and Shangshantou in Jingning She Autonomous county (Figure 1).

\subsection{Acquisition and Processing of Climate and Altitude Data}

The climate and altitude data utilized in this study were sourced from WorldClim v2.0 (https: //worldclim.org/) [22], and the highest spatial resolution level of 30" was selected. As this study aimed to predict the potential distribution area of $C$. tientaiensis in China, the whole of China was taken as the background for model prediction. Climate factors were selected for three time periods: current (1970-2000), the 2050s (2040-2060), and the 2070s (2060-2080). The climate data for each period included 19 climate factors (Table S2). The data for future climate scenarios were selected from the general climate system model community climate system model version 4 (CCSM4), which included four representative concentration pathways (RCPs) for greenhouse gases: RCP 2.6, RCP 4.5, RCP 6.0, and RCP $8.5[22,23]$.

Twenty BIOCLIM variables were initially used for model establishment in this study (Table S2). In order to minimize any inaccuracy in model prediction due to the correlation between climate variables, climate factors with no contribution rate to model prediction were then excluded. Fourteen climate and one altitude variables were selected to build the final model (Table 1).

Table 1. Contribution of the 15 BIOCLIM variables in the MaxEnt modeling (\%).

\begin{tabular}{cccc}
\hline Variable & Variable Description & Unit & Contribution Rate \\
\hline bio17 & Precipitation of the driest quarter & $\mathrm{mm}$ & 17.7 \\
bio15 & CV of precipitation seasonality & $\%$ & 16.6 \\
bio12 & Annual precipitation & $\mathrm{mm}$ & 15.3 \\
bio14 & Precipitation of the driest month & $\mathrm{mm}$ & 13.0 \\
bio18 & Precipitation of the coldest quarter & $\mathrm{mm}$ & 10.7 \\
\hline
\end{tabular}


Table 1. Cont.

\begin{tabular}{cccc}
\hline Variable & Variable Description & Unit & Contribution Rate \\
\hline bio3 & Isothermality (bio2/bio7 $\times 100)$ & $\%$ & 9.7 \\
bio2 & Mean diurnal temperature range & ${ }^{\circ} \mathrm{C}$ & 9.5 \\
bio10 & Mean temperature of warmest quarter & ${ }^{\circ} \mathrm{C}$ & 3.5 \\
bio1 & Annual mean temperature & ${ }^{\circ} \mathrm{C}$ & 1.5 \\
bio19 & Precipitation of the coldest quarter & $\mathrm{mm}$ & 1.0 \\
bio8 & Mean temperature of wettest quarter & ${ }^{\circ} \mathrm{C}$ & 0.8 \\
alt & Altitude & $\mathrm{m}$ & 0.3 \\
bio16 & Precipitation of wettest quarter & $\mathrm{mm}$ & 0.2 \\
bio9 & Mean temperature of driest quarter & ${ }^{\circ} \mathrm{C}$ & 0.1 \\
bio6 & Min temperature of coldest month & ${ }^{\circ} \mathrm{C}$ & 0.0 \\
\hline
\end{tabular}

\subsection{MaxEnt Model Operation and Evaluation}

The MaxEnt 3.4.0 software (http://biodiversityinformatics.amnh.org/open_source/maxent/) [14] was used to project the niches of $C$. tientaiensis in this study. The distribution data for $C$. tientaiensis, climate factors, and altitude data were imported into the MaxEnt 3.4.0 software. In this study, $25 \%$ of the distribution points were selected as the test set, and $75 \%$ of the distribution points were utilized as a training set for the MaxEnt model [24]. Jackknifing was used to check the weights of the environmental variables, the output format was logistic, and cross-validation was used as the replicated run type. Since the choice of feature combinations is related to the number of species distribution points $[25,26]$, linear feature, quadratic feature and hinge feature combinations were selected. Other parameters included a maximum of 10,000 background points, a maximum of 500 iterations, a convergence threshold of 0.00001 , and a regularization value of 1 .

The area under the curve (AUC) value is often used to evaluate the accuracy of models $[27,28]$. Since the MaxEnt model is unable to produce unique solutions [29], we used an average value to get an average prediction result. The model was run nine times, and the average of the results from the nine runs was used as the final prediction result. The MaxEnt model also provides a variety of ways to evaluate the importance of environmental variables on species distributions. In this study, the contribution rate was used to evaluate the dominant factors affecting the geographical distributions of $C$. tientaiensis.

\subsection{Classification of Habitat Suitability}

The final prediction results were imported into ArcGIS 10.2 (ESRI Inc., California, USA), and the potential distribution map of $C$. tientaiensis was drawn after the prediction results were reclassified. In order to indicate whether the study area is suitable for the survival and distribution of species, the MaxEnt model provides a corresponding suitability index, ranging from 0 to 1 , for the prediction results. Generally, the higher the suitability index of the study area, the more suitable the area is for species survival and distribution.

The thresholds of "equate entropy of thresholded and original distributions" and " 10 percentile presence in the training data" were used as the thresholds of suitable distribution areas and core suitable areas, respectively. We used these values combined with equal intervals to divide the suitability index into five grades: $0.0000-0.0746,0.0746-0.2978,0.2978-0.5210,0.5210-0.7605$, and $0.7605-1.0000$. Areas with a suitability index between 0 and 0.0746 were defined as unsuitable areas, areas between 0.0746 and 1 were suitable distribution areas, areas between 0.0746 and 0.521 were generally suitable areas, and areas between 0.521 and 1 were core suitable areas.

\subsection{Dynamic Changes and Centroid Migrations of the Suitable Distribution Area}

The raster calculator tool in ArcGIS 10.2 was used to extract the regions that were suitable distribution areas for C. tientaiensis under both the current and future climate scenarios. In this study, 
the areas that were determined to be suitable in both the current and future climate scenarios were defined as "Stable.", while areas that are currently suitable but are not suitable in future climate scenarios were defined as "Lost," and areas that are currently unsuitable but are suitable in the future were defined as "Increased." At the same time, we identified places that were suitable distribution areas for C. tientaiensis in any of the current, 2050s and 2070s climates, to further assess the suitability of certain areas for conservation.

The distribution of suitable distribution areas of $C$. tientaiensis were scattered and their outline was irregular. To visually indicate the changing trends of the suitable distribution areas of $C$. tientaiensis, ArcGIS 10.2 was used to calculate the centroid of the current and future suitable distribution areas of $C$. tientaiensis. The centroid can reduce the suitable distribution area of the species to a point, which indicates the direction of the change in suitable distribution areas over time [30].

\section{Results}

\subsection{Model Accuracy}

Under the current climatic conditions, the average AUC value output of the MaxEnt model, after being run nine times, was 0.999 (Figure 2); this shows that the prediction accuracy of the current geographic distribution was extremely high. The prediction results can thus be used to accurately identify the geographical distributions and potential distributions of $C$. tientaiensis in China.

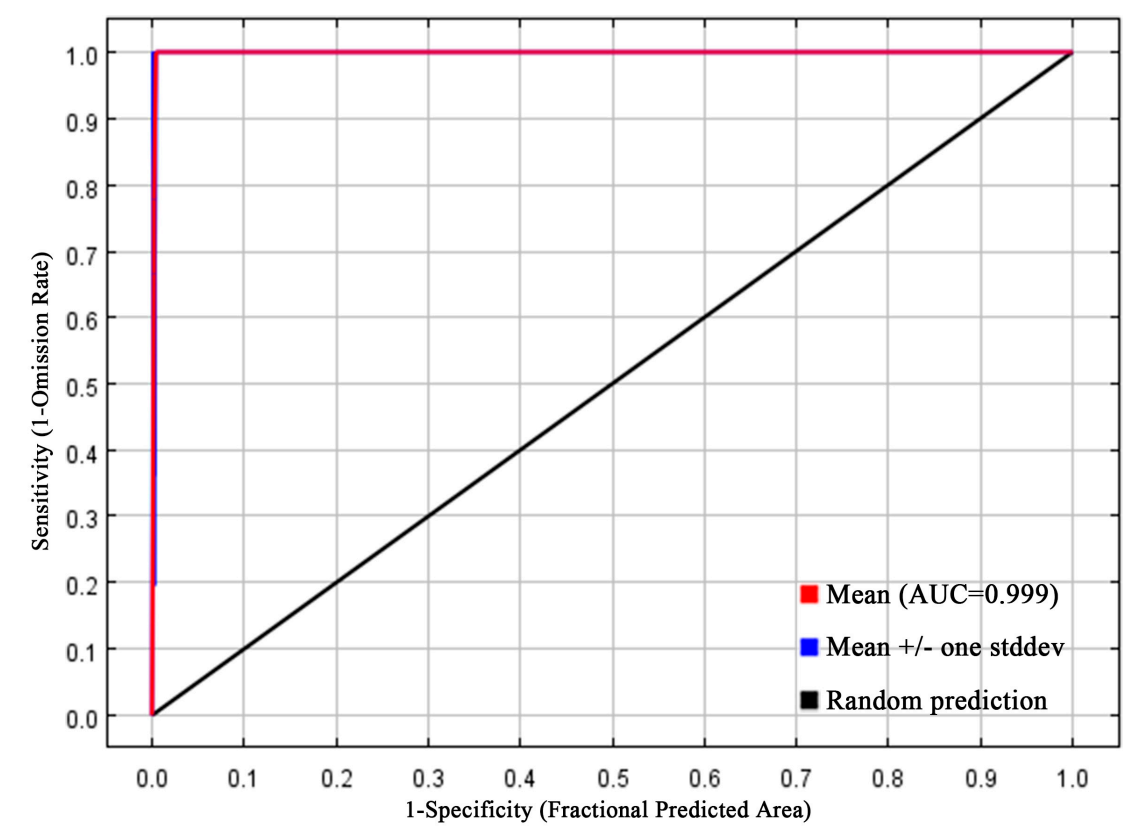

Figure 2. Prediction validation with receiver operator characteristic (ROC) curves using the MaxEnt model.

\subsection{Suitable Distribution Areas of $C$. tientaiensis in the Current Climate}

At present, C. tientaiensis is only known to have a narrow distribution area in the mountain forests in the east and south of Zhejiang province, with very few natural populations. Based on the 58 distribution data points (Table S1) and 15 BIOCLIM variables (Table 1), the current suitable distribution areas for C. tientaiensis in China were predicted (Figure 3). The suitable distribution area for C. tientaiensis in China was determined to be approximately $90.79 \times 10^{3} \mathrm{~km}^{2}$, accounting for only $0.94 \%$ of China's land area. Furthermore, the core suitable area was approximately $13.65 \times 10^{3} \mathrm{~km}^{2}$, accounting for only $0.14 \%$ of China's land area. 


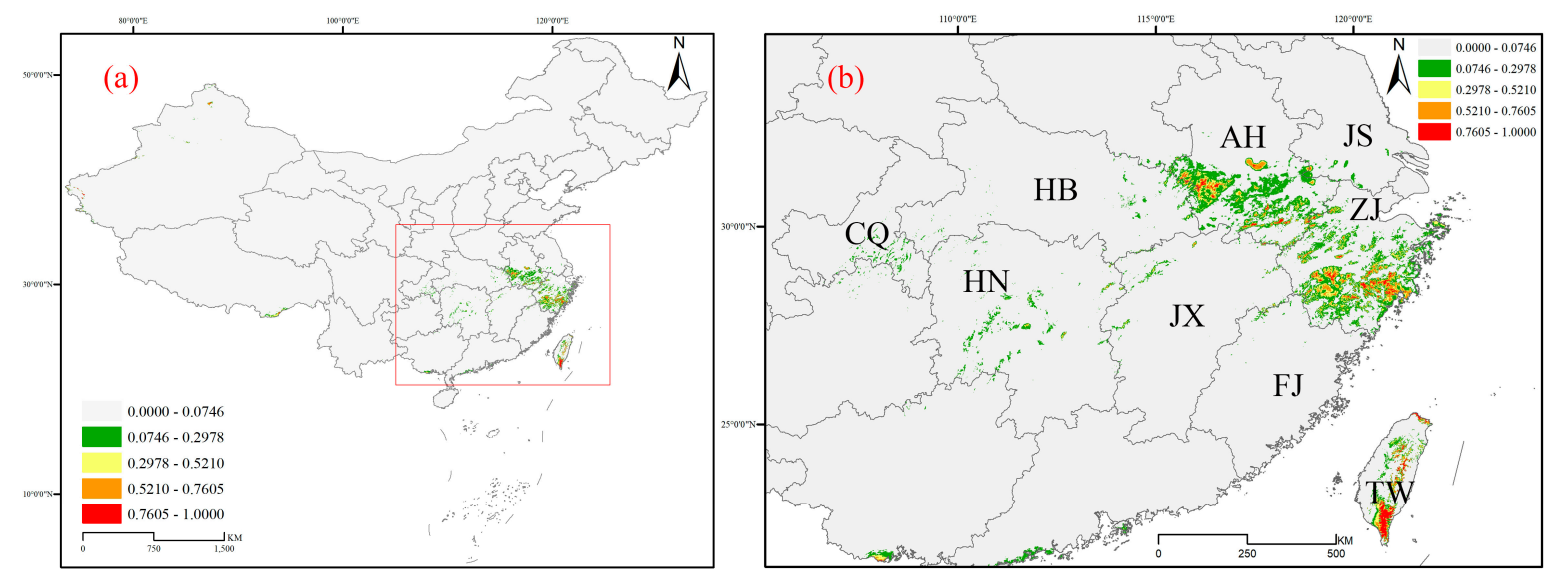

Figure 3. Suitable distribution areas for Carpinus tientaiensis in China under current climatic conditions. (a) Across all of China; (b) In the east of China. ZJ: Zhejiang Province; JS: Jiangsu Province; AH: Anhui Province; HB: Hubei Province; HN: Hunan Province; CQ: Chongqing City; JX: Jiangxi Province; FJ: Fujian Province; TW: Taiwan Province.

The results showed that there were suitable distribution areas for $C$. tientaiensis in Taiwan province and southern Anhui in addition to Zhejiang. The suitable distribution areas were mainly located in the eastern parts of China, including the mountains in south Zhejiang, the Dabie mountains in the southwest of Anhui, the Jiuhua and Huangshan mountains in the southeast of Anhui, and the central and southern mountains in Taiwan province (Figure 3). There were some scattered suitable distribution areas in Jiangxi, Hunan, Chongqing, and other places, while other areas in China were unsuitable for the survival and distribution of $C$. tientaiensis. Core suitable areas were concentrated in the Xianxialing and Kuocang mountains in south Zhejiang, and the central and southern mountains of Taiwan, with some other areas in the Dabie mountains, and Jiuhua mountains in the southeast of Anhui (Figure 3).

\subsection{Dominant Environmental Factors Limiting the Survival and Distribution of C. tientaiensis}

According to the contribution rate of the variables in the MaxEnt model output (Table 1), many of the selected climate factors played an important role in the model, and some precipitation factors had leading roles in the survival and distribution of $C$. tientaiensis. Among these, the precipitation of the driest quarter (bio17), coefficient of variation (CV) of precipitation seasonality (bio15), annual precipitation (bio12), precipitation of the driest month (bio14), precipitation of the coldest quarter (bio18), and isothermality (bio3) played major roles in the distribution of C. tientaiensis, with a cumulative contribution of $83 \%$.

In this study, regions with a suitability index $\geq 0.521$ were regarded as core suitable regions for C. tientaiensis. Therefore, when the distribution probability was $\geq 0.521$, the corresponding environmental factor values were suitable for the survival and distribution of $C$. tientaiensis, and these values limited the distribution regions of $C$. tientaiensis. According to the contribution rate (Table 1) and response curve (Figure 4), the precipitation of the driest quarter (bio17) had the greatest impact on the survival and distribution of $C$. tientaiensis. When the precipitation of the driest quarter (bio17) was more than $700 \mathrm{~mm}$, the probability of the occurrence of $C$. tientaiensis peaked. If the probability of the occurrence was $\geq 0.521$, the adaptation range of the precipitation of the driest quarter (bio17) was about 190-700 mm. In addition, if the probability of the existence was $\geq 0.521$, the adaptive range of the CV of precipitation seasonality (bio15), annual precipitation (bio12), precipitation of the driest month (bio14), precipitation of the coldest quarter (bio18), and isothermality (bio3) were approximately 2.2-5.1\%, 1900-4500, 48-180, 750-1800 mm, and 16-26\%, respectively. 

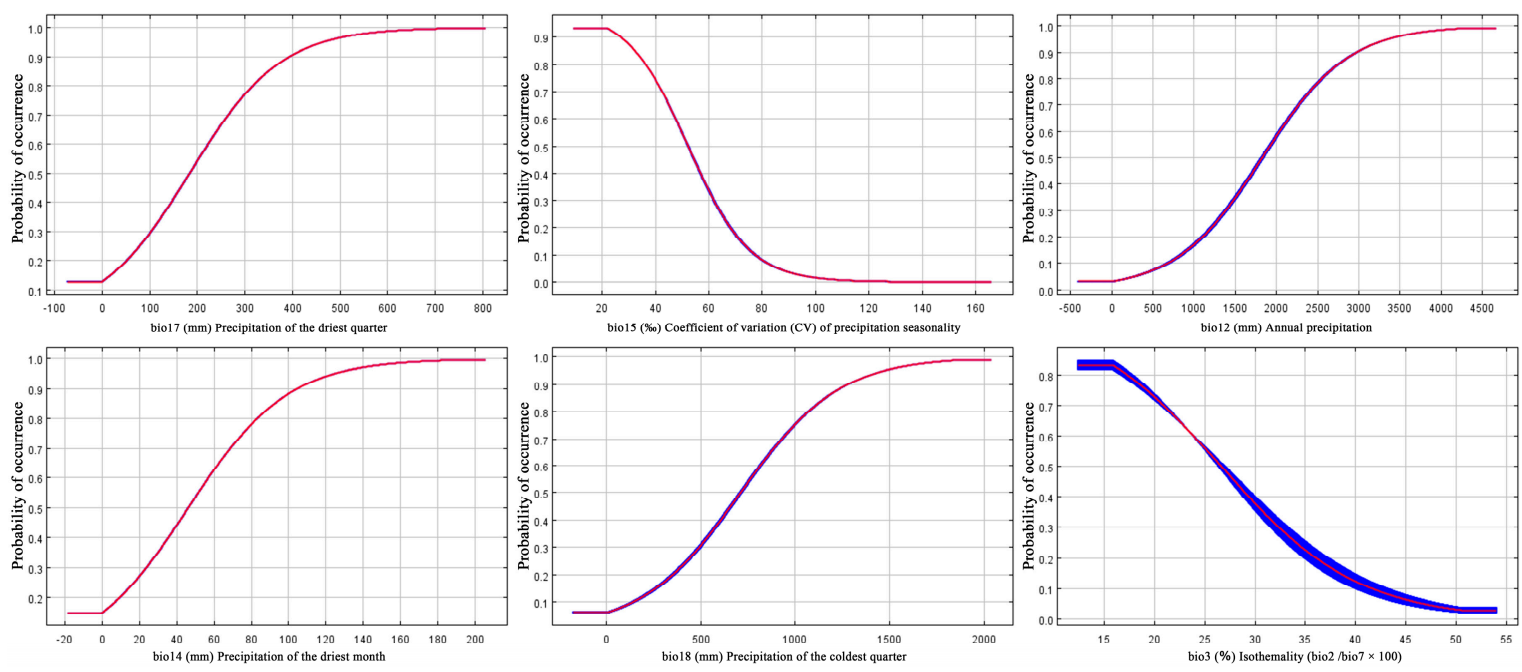

Figure 4. Response curves of the major climate factors.

\subsection{Suitable Distribution Areas of C. tientaiensis in Future Climate Change Scenarios}

Compared with the current situation, in future climate change scenarios, the suitable distribution areas will decrease by $71 \%$ and the core suitable area will decrease by $81 \%$ (Figures 3 and 5, Table 2). The areas of suitable distribution were the smallest in the scenario with RCP 6.0 in the 2070s, and this was $79 \%$ less than the current suitable distribution area. RCP 8.5 in the 2050s, resulted in the largest suitable distribution area, but it was still $64 \%$ lower than it is today. The smallest amount of core suitable areas-85\% lower than the current situation-was found under RCP 4.5 in the 2050s. Although the area of the RCP 8.5 in the 2050s was the largest of the scenarios, it was still $73 \%$ lower when compared with current conditions.

Table 2. The suitable distribution and core distribution areas of Carpinus tientaiensis and the changes in the suitable distribution area under future climate change scenarios.

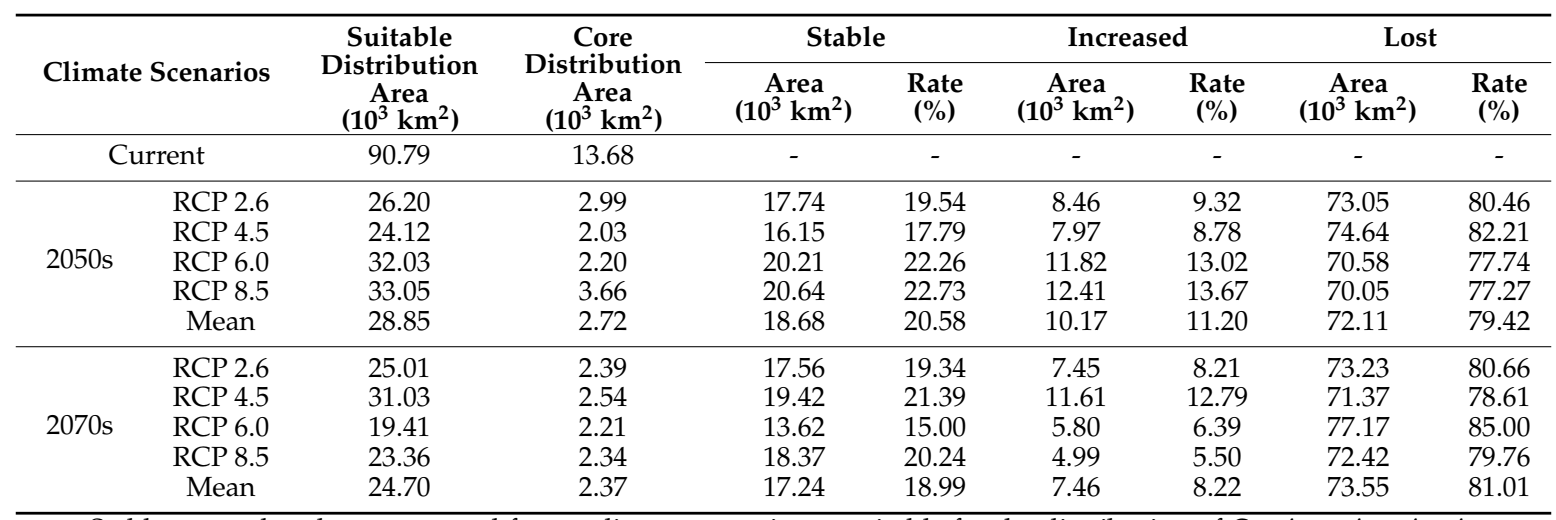

Stable, areas that the current and future climate scenario are suitable for the distribution of Carpinus tientaiensis; Increased, areas that are currently unsuitable but are suitable in the future; Lost, areas that are currently suitable but are not suitable in future climate scenarios. Rate $=($ area of Stable or Increased or Lost/current area $) \times 100 \%$.

The suitable distribution areas of $C$. tientaiensis were obviously reduced and tended to move slightly north and obviously eastward (Figures 3 and 5). The suitable distribution area in Zhejiang was much reduced compared with the current scenario, and was mainly located in the Kuocang mountains in southern Zhejiang. The suitable distribution areas in southern Taiwan were almost completely lost, and only a few suitable distribution areas were preserved in central and north Taiwan. However, a small number of suitable distribution areas began to appear around the Qinling and Daba mountains in the east of Hubei. There was also a significant reduction in core suitable areas. In the southern part of Zhejiang, Anhui and Taiwan, the core suitable areas were almost completely lost. The remaining core suitable areas were mainly located in the Kuocang mountains in south Zhejiang and central Taiwan. 

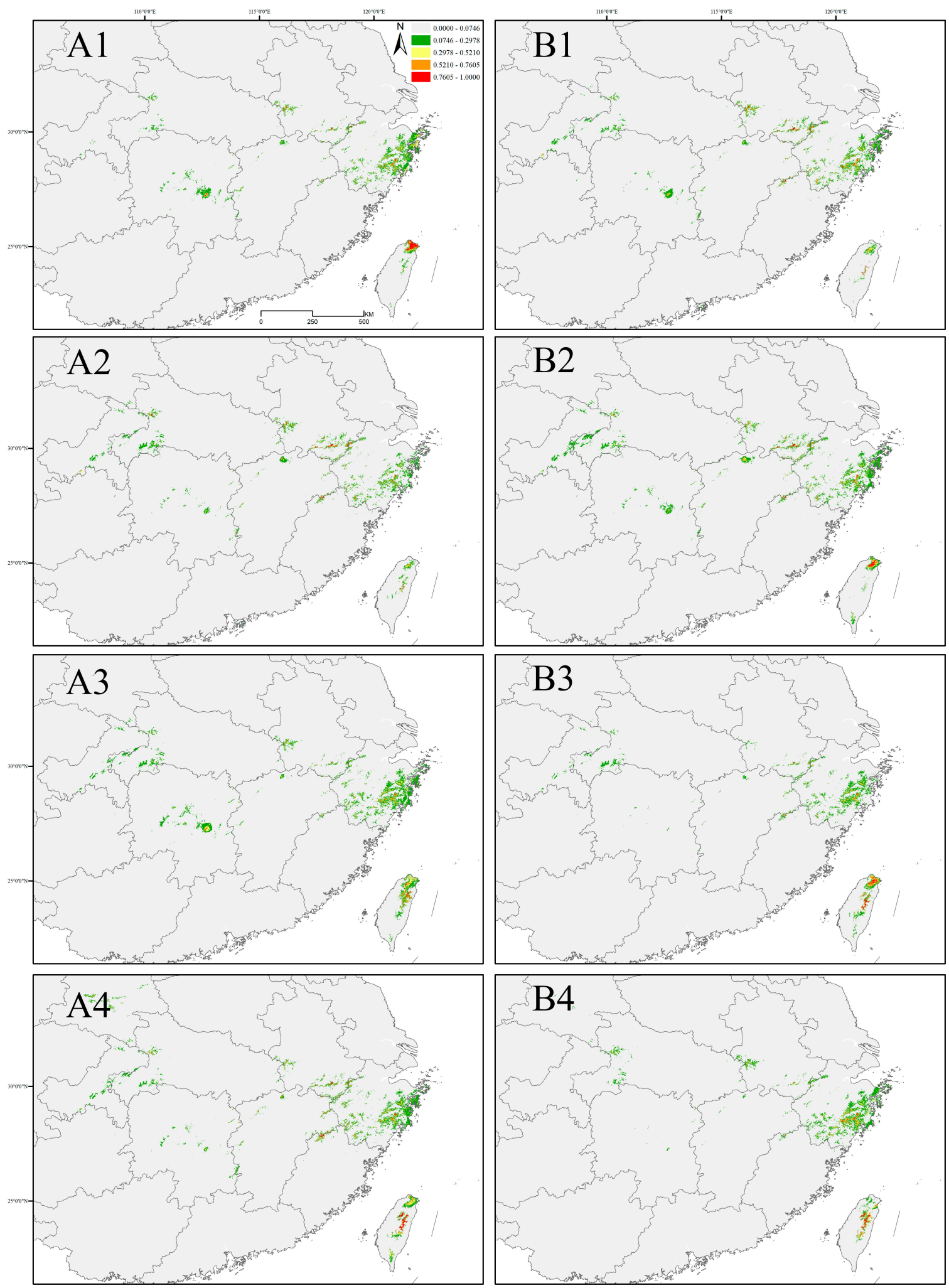

Figure 5. Suitable distribution areas of Carpinus tientaiensis in future climate change scenarios. (A1-A4), the 2050s; (B1-B4), the 2070s; 1, future climate scenario RCP 2.6; 2, future climate scenario RCP 4.5; 3 , future climate scenario RCP 6.0; 4, future climate scenario RCP 8.5.

Compared with the current situation, the spatial locations for the suitable distribution areas of $C$. tientaiensis were shown to change markedly in the future scenarios (Figure 6, Table 2). In the 2050s and 2070s climate scenarios, only $18 \%$ of the suitable distribution areas were relatively stable, 
which has a strong buffer capacity against climate change. However, $80 \%$ of the suitable distribution areas were vulnerable to climate impacts and were lost in the climate change scenarios. There were few opportunities for expansion under the future climate change scenarios, as only about $10 \%$ of the unsuitable area became suitable. In Zhejiang in the future scenarios, the loss of suitable distribution areas of $C$. tientaiensis was significant, and it appeared for the most part that the west of Zhejiang would no longer be suitable for the survival of $C$. tientaiensis. Kuocang mountain in south Zhejiang and the mountain areas in central Taiwan province were found to preserve many suitable distribution areas in the future.

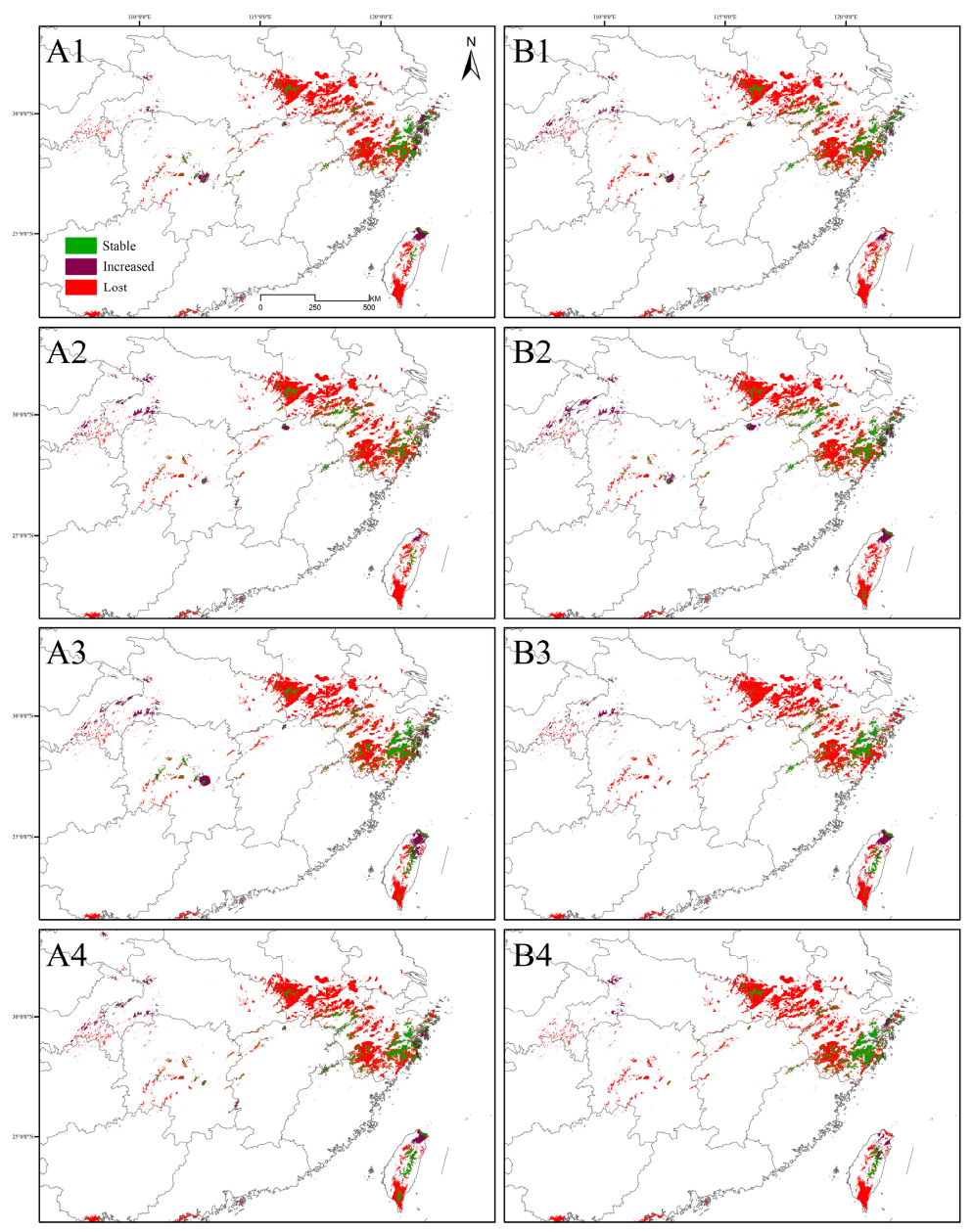

Figure 6. Dynamic changes of the suitable distribution area of Carpinus tientaiensis under current and future climate scenarios. (A1-A4), 2050s; (B1-B4), 2070s; 1, future climate scenario RCP 2.6; 2, future climate scenario RCP 4.5; 3, future climate scenario RCP 6.0; 4, future climate scenario RCP 8.5; Stable, areas that the current and future climate scenarios are suitable for the distribution of Carpinus tientaiensis; Increased, areas that are currently unsuitable but are suitable in the future; Lost, areas that are currently suitable but are not suitable in future climate scenarios.

Xianxialing in the southwest of Zhejiang province, the lower altitudes in south Anhui, and the lower altitudes in south and central Taiwan, were all vulnerable to the impacts of climate change. In the future, there will be a significant loss of the original suitable distribution areas because of climate change. In addition, the scattered suitable distribution areas in Jiangxi, Chongqing and Hunan were found to be almost completely lost. However, it is worth noting that with the future climate change scenarios, there are some suitable distribution areas in the mountainous areas of Hubei and Chongqing as well as in the Snow mountains in north Taiwan (Figure 6). At the same time, Kuocang, Dapan and Tiantai mountains in south Zhejiang, high altitude areas of Dabie mountain in the southwest of Anhui, 
and Jiuhua mountain in the southeast of Anhui, were relatively stable, and suitable for the survival of C. tientaiensis in the future climate change scenarios. These regions may be appropriate sites for ex situ conservation or introduction of $C$. tientaiensis (Figure 7).

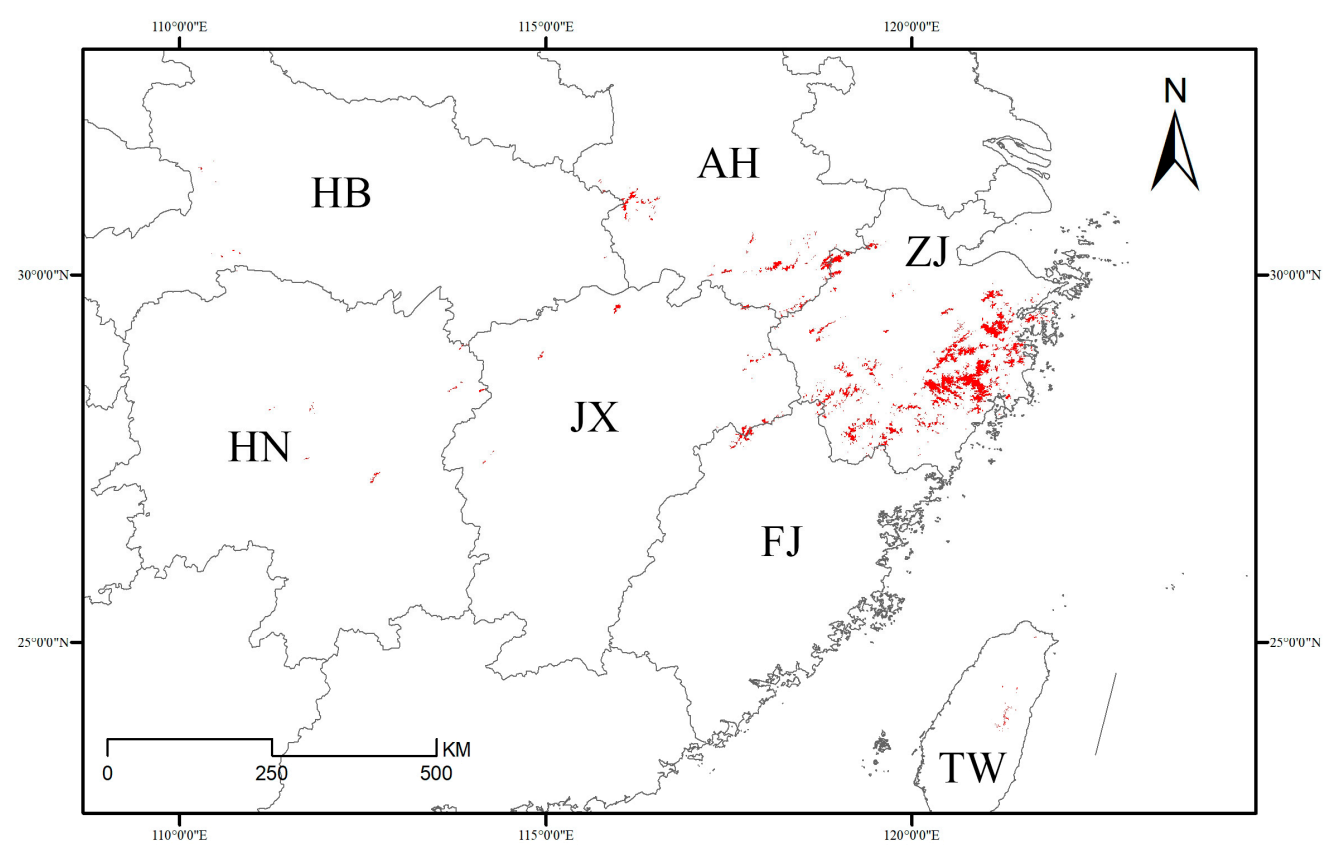

Figure 7. Suitable distribution areas of Carpinus tientaiensis in current and future scenarios. The red area indicates locations where the current, 2050s and 2070s climate scenarios are all suitable for C. tientaiensis. ZJ: Zhejiang Province; AH: Anhui Province; JX: Jiangxi Province; FJ: Fujian Province; HB: Hubei Province; HN: Hunan Province; TW: Taiwan Province.

Suitable distribution areas for $C$. tientaiensis are relatively scattered, and so this study analyzed the spatial changes of its geographical distribution through the changes in the centroids from the suitable distribution areas, in different climate scenarios. In the 2050s and 2070s, the centroid generally moved to the east (Figure 8).

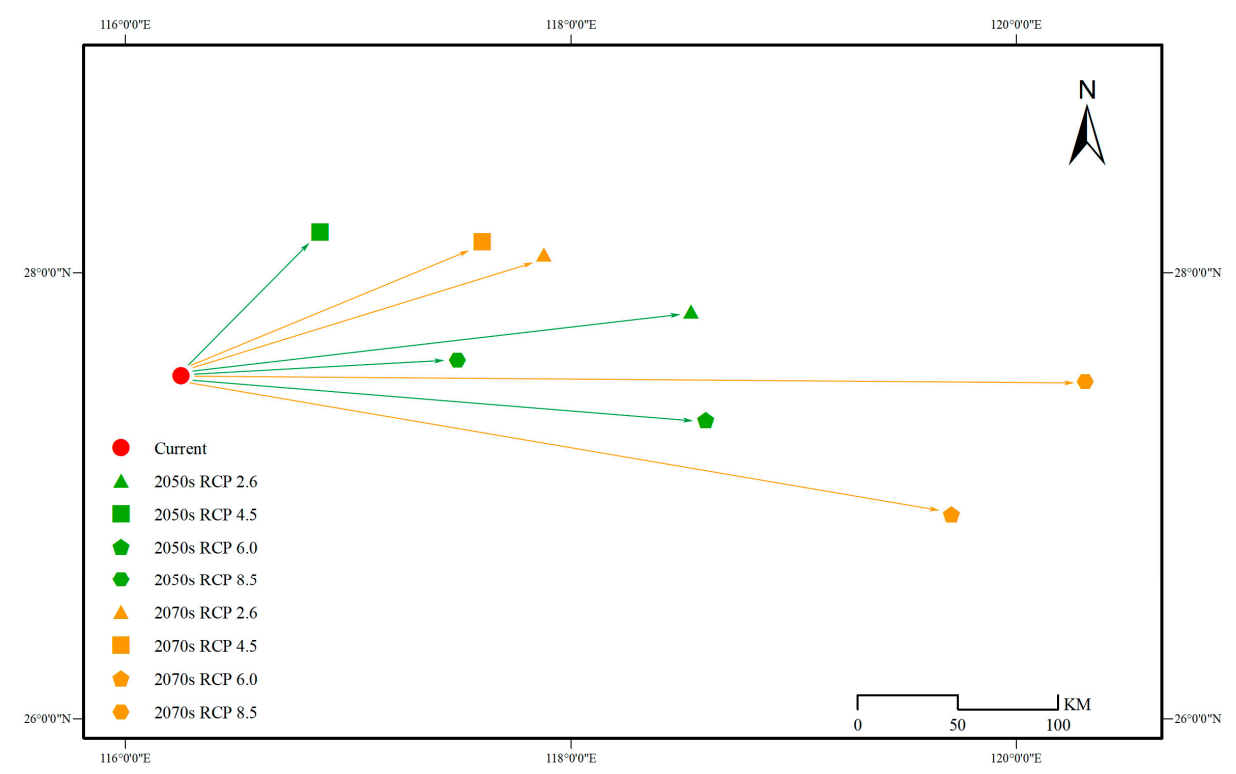

Figure 8. Centroid migration in the suitable distribution area of Carpinus tientaiensis under different climate scenarios. The arrow indicates the direction of change of the centroid of the suitable distribution area with time. 


\section{Discussion}

There is some debate over the use of correlative models instead of mechanistic models to study the potential distribution of species, because some researchers argue that the correlation between the species and environment may cease to exist or may change in future decades. It has also been suggested that, for this reason, correlative models will provide more robust results compared to mechanistic models under future climate scenarios [31,32]. However, despite their usefulness, mechanistic models are hard to build, time consuming, and they require a good knowledge and background information about target species [31,32]. Many SDMs have been established in the world to predict species distribution areas, but few models can be effectively used for endangered species and rare species with limited distribution data [33]. As most SDMs are sensitive to sample size [34] and require large amounts of distribution data [35], they cannot accurately predict the potential distribution of endangered species. However, the MaxEnt model is a powerful tool for predicting potential distribution for endangered species with a narrow distribution range or limited distribution data [14,34,36,37]. Even in the case of extremely limited species distribution data, the accuracy is high $[37,38]$. Therefore, the MaxEnt model was selected in this study.

In many previous studies on the potential distribution of plants, although researchers have recognized that other factors, such as soil, may play a role in species distribution, they have often focused only on the role of the climate on the potential distribution of plants. In the early stage of this study, we tried to add soil data, provided by China soil map based harmonized world soil database v1.1 (http://westdc.westgis.ac.cn), into the environmental variables, but the prediction results using soil data were not reasonable. Therefore, this study did not use soil data for modeling. There may not yet be an effective method to incorporate soil data better in species distribution models. In future research, working out how to use soil data effectively to study the potential distribution of species will be a significant research topic.

The distribution and richness of many species will be impacted by climate change [39], and climate change may increase the risk of species extinction [7]. Thomas et al. predicted that $15-37 \%$ of species in their research sample "commit to extinction," according to mid-range climate-warming scenarios for 2050 [39]. Iverson et al. predicted that of 134 tree species in the eastern United States, 54 would lose at least $10 \%$ of their suitable habitats under climate change compared with the current [40]. Climate change has resulted in more severe challenges for the survival and distribution of endangered species. There are about 50 species of Carpinus and 33 of these species are recorded in the Flora of China, of which 27 are endemic to China, with relatively narrow distributions [19]. There are many endangered species of Carpinus, such as C. tientaiensis [20], C. putoensis [41], C. hebestroma [42], and C. langaoensis [43], all of which have relatively narrow distributions in only one or several places. The aim of this investigation was to study the impacts of climate change on C. tientaiensis, a typical endemic and endangered species, and to develop effective conservation strategies that would be of great significance for the study and protection of other endemic species of Carpinus as well as other endangered species.

The prediction results showed that the suitable distribution area of $C$. tientaiensis was small, but there were many suitable distribution areas in the Xianxialing and Kuocang mountains in the south of Zhejiang Province. C. tientaiensis was first found on Tiantai mountain in Zhejiang province, but in recent years, it has also been found on Dapan mountain, Yangtianhe, and Shangtoutou. At present, it is possible that some wild communities have still not yet been identified. As early as the beginning of the 21st century, Engler et al. (2004) facilitated the use of model predictions to suggest new sampling sites for endangered species [44]. In line with this, our modeling has shown that the Xianxialing and Kuocang mountains should be sites for future field investigations to search for new wild populations of $C$. tientaiensis. From the contribution rates of each climate variable in the MaxEnt modeling (Table 1), it was determined that the climatic conditions limited the survival and distribution of $C$. tientaiensis. The precipitation of the driest quarter (bio17) seriously affected the survival and distribution of C. tientaiensis, which indicated that it had strict requirements for its water conditions, especially during the dry season. There is abundant rainfall in Zhejiang Province, which indicates that $C$. tientaiensis 
is a species adapted to wet conditions. The response curve also shows that the adaptive range of C. tientaiensis for the precipitation of the driest quarter (bio17) was about 220-700 $\mathrm{mm}$, and high rainfall and waterlogging environments could also affect its survival.

C. tientaiensis is only distributed in Zhejiang Province, which is near the East China Sea. In recent decades, as a consequence of global climate change, the warming rate in this region has been five times that of the global average [45], indicating that it is a region that is highly sensitive to climate change. Consequently, because of future climate change, the suitable distribution areas for C. tientaiensis will be significantly reduced, or almost completely lost. Generally, when the climate warms, species distributions move toward high-latitude or -altitude areas [46]. With future climate change, only Kuocang, Dapan and Tiantai Mountains in the southeast of Zhejiang, and part of the high-altitude areas in the south of Anhui and the central part of Taiwan will have suitable areas for C. tientaiensis, and the low-altitude suitable areas will be almost completely lost. In addition, the suitable distribution area of $C$. tientaiensis showed an obviously shift to the north, and the suitable distribution area in the south of Taiwan was almost completely lost. Nearly all the suitable distribution areas in the southwest of Anhui and the west of Zhejiang were also obviously lost, which explains why the centroid of the suitable distribution areas of $C$. tientaiensis, moved to the east in the future. However, compared with the eastern part of China, the climate in the center changes only gradually from the north to the south with a strong buffer capacity and, consequently, the impact of climate change on this region was small [47]. Therefore, in the 2070s scenario, while the suitable distribution area of $C$. tientaiensis was predicted to be lost from the eastern part of China, in the Qinling and Daba mountains in Hubei and other areas of China, some suitable distribution areas appeared.

While climate change has serious impacts on species habitats [3], human activities, such as tourism, can also result in their loss [48], and in recent years they have had an increasing impact [49]. The construction of reservoirs and dams has also led to a reduction in species richness, habitat fragmentation, and even disappearance [50-52]. At present, among the four communities of C. tientaiensis, only Dapan mountain is a National Nature Reserve, and it has not yet developed its tourism industry. Tiantai mountain, however, is a popular tourist destination, and there is also a reservoir and dam at Yangtianhe, and Shangshantou that attracts tourists because of its large Rhododendron fortunei, resulting in a serious impact on the natural habitat of $C$. tientaiensis. In order to prevent the disappearance of $C$. tientaiensis from natural communities, more attention should be given to reducing human disturbance and the establishment of natural reserves at its sites of origin.

Carpinus is a wind-pollinated plant [53] and, specifically, the rainy climate in the spring and summer of Zhejiang province has serious impacts on the pollination of $C$. tientaiensis. In years with abundant rainfall and high wind speed, $C$. tientaiensis bears little fruit. In addition, the different maturation times of the $C$. tientaiensis pistil and stamen have serious negative effects on the pollination rate and seed setting rate. This means that the natural regeneration ability of the $C$. tientaiensis community is weak and can result in a decreasing population. Consequently, it is necessary to expand the population base by artificial breeding based on the existing communities. An important means for the protection of endangered species is to advocate for their introduction and cultivation in botanical gardens. This is because there is a high level of artificial management in botanical gardens [54], and the management of wild species is conducted scientifically, with the management personnel being highly trained [49]. At the same time, introduction and cultivation in botanical gardens should be actively carried out as high-intensity artificial management practices.

C. tientaiensis is a slow-growing and long-life tree species, its natural life span can reach hundreds of years. In this study, we focused on finding potential distribution areas of $C$. tientaiensis and assessing the impact of future climate change on its survival and distribution. The suitable distribution areas of C. tientaiensis were identified from present to 2070s, which provided a reference for field investigation and ex situ conservation of $C$. tientaiensis. However, it was not clear what changes will occur in the survival and distribution of C. tientaiensis after 2070s. However, due to the lack of long-term 
environmental data, we were unable to carry out more studies to assess the survival and distribution of C. tientaiensis after 2070s.

\section{Conclusions}

By utilizing the MaxEnt model and ArcGIS software, this study constructed the potential current and future distributions of $C$. tientaiensis. The current suitable distribution areas for $C$. tientaiensis were mainly located in eastern China in south Zhejiang and Anhui provinces, and the center and south of Taiwan Province. The core suitable areas were mainly located in the Xianxialing and Kuocang Mountains in south Zhejiang and center and south of Taiwan province. The survival and distribution of $C$. tientaiensis was limited by climatic factors such as the precipitation of the driest quarter (bio17), CV of precipitation seasonality (bio15), annual precipitation (bio12), precipitation of the driest month (bio14), precipitation of the coldest quarter (bio18), and isothermality (bio3).

Endangered plants have important scientific and ecological value, and it is thus important to seek scientific protection strategies for them. The mountain areas of Xianxialing and Kuocang, in south Zhejiang, will be the focus of future field investigations, as it is possible that wild communities of C. tientaiensis that have not been previously identified exist in these areas. Furthermore, the Kuocang, Dabie, and Jiuhua mountains were found to be suitable for the introduction, cultivation, and ex situ conservation of $C$. tientaiensis.

In the future, most of the suitable distribution areas and core suitable areas for $C$. tientaiensis will be lost. Furthermore, with climate change, most of the suitable distribution areas for $C$. tientaiensis will no longer be suitable, which highlights the current need for their protection and management. As $C$. tientaiensis is evidently going to be threatened by climate change, we should strengthen the conservation and management strategies in its original habitats, and carry out artificial cultivation in these areas to expand the population and enhance the ability of the natural populations to cope with climate change. At the same time, ex situ conservation and cultivations in botanical gardens should be actively carried out, as their high-intensity artificial management practices will aid the conservation of C. tientaiensis.

Supplementary Materials: The following are available online at http://www.mdpi.com/1999-4907/11/7/774/s1, Table S1: Distribution data of Carpinus tientaiensis, Table S2: Contribution of the 20 BIOCLIM variables in the MaxEnt modeling (\%).

Author Contributions: Conceptualization, R.Z. and Z.Z.; methodology, R.Z.; validation, R.Z., Y.T. and M.S.; formal analysis, R.Z. and X.C.; investigation, R.Z. and Q.H.; data curation, R.Z. and X.C.; writing-original draft preparation, R.Z.; writing-review and editing, R.Z., X.C., Q.H. and Z.Z.; supervision, Z.Z.; funding acquisition, Z.Z. All authors have read and agreed to the published version of the manuscript.

Funding: This research was funded by the Forestry and Grassland Science and Technology Achievements Promotion Project of National Forestry and Grassland Administration (2019133119); and the Fifth Stage Funded Research Projects of 333 in Jiangsu Province (BRA2018065) and the National Natural Science Foundation of China (31770752).

Conflicts of Interest: The authors declare no conflict of interest.

\section{References}

1. Manthey, M.; Box, E.O. Realized climatic niches of deciduous trees: Comparing western Eurasia and eastern North America. J. Biogeogr. 2007, 34, 1028-1040. [CrossRef]

2. Intergovernmental Panel on Climate Change. Climate Change 2013: The Physical Science Basis: Working Group I Contribution to the Fifth Assessment Report of the Intergovernmental Panel on Climate Change; Cambridge University Press: Cambridge, UK, 2014. [CrossRef]

3. Barrett, M.A.; Brown, J.L.; Junge, R.E.; Yoder, A.D. Climate change, predictive modeling and lemur health: Assessing impacts of changing climate on health and conservation in madagascar. Biol. Conserv. 2013, 157, 409-422. [CrossRef] 
4. Bertrand, R.; Lenoir, J.; Piedallu, C.; Riofrio-Dillon, G.; Ruffray, P.; Vidal, C.; Pierrat, J.; Gégout, J. Changes in plant community composition lag behind warming in lowland forests. Nature 2011, 479, 517-520. [CrossRef] [PubMed]

5. Dyderski, M.K.; Paź-Dyderska, S.; Frelich, L.E.; Jagodziński, A.M. How much does climate change threaten European forest tree species distributions? Glob. Chang. Biol. 2018, 24, 1150-1163. [CrossRef]

6. Bellard, C.; Bertelsmeier, C.; Leadley, P.; Thuiller, W.; Courchamp, F. Impacts of climate change on the future of biodiversity. Ecol. Lett. 2012, 15, 365-377. [CrossRef]

7. Urban, M.C. Climate change. Accelerating extinction risk from climate change. Science 2015, 348, 571-573. [CrossRef]

8. Heinrichs, J.A.; Bender, D.J.; Schumaker, N.H. Habitat degradation and loss as key drivers of regional population extinction. Ecol. Model. 2016, 335, 64-73. [CrossRef]

9. Bogich, T.L.; Barker, G.M.; Mahlfeld, K.; Climo, F.; Green, R.; Balmford, A. Fragmentation, grazing and the species-area relationship. Ecography 2012, 35, 224-231. [CrossRef]

10. Peterson, A.T.; Soberón, J.; Pearson, R.G.; Anderson, R.P.; Martínez-Meyer, E.; Nakamura, M.; Araújo, M. Ecological Niches and Geographic Distributions; Princeton University Press: Princeton, NJ, USA, 2011.

11. Qin, A.L.; Liu, B.; Guo, Q.S.; Bussmann, R.W.; Ma, F.Q.; Jian, Z.J.; Xu, G.X.; Pei, S.X. Maxent modeling for predicting impacts of climate change on the potential distribution of Thuja sutchuenensis Franch., an extremely endangered conifer from southwestern China. Glob. Ecol. Conserv. 2017, 10, 139-146. [CrossRef]

12. Newbold, T. Applications and limitations of museum data for conservation and ecology, with particular attention to species distribution models. Prog. Phys. Geogr. 2010, 34, 3-22. [CrossRef]

13. Marcer, A.; Sáez, L.; Molowny-Horas, R.; Pons, X.; Pino, J. Using species distribution modelling to disentangle realised versus potential distributions for rare species conservation. Biol. Conserv. 2013, 166, 221-230. [CrossRef]

14. Phillips, S.J.; Anderson, R.P.; Schapire, R.E. Maximum entropy modeling of species geographic distribution. Ecol. Model. 2006, 19, 231-259. [CrossRef]

15. Li, G.Q.; Du, S.; Wen, Z.M. Mapping the climatic suitable habitat of oriental arborvitae (Platycladus orientalis) for introduction and cultivation at a global scale. Sci. Rep. 2016, 6, 30009. [CrossRef] [PubMed]

16. Borthakur, S.K.; Baruah, P.S.; Deka, K.; Das, P.; Sarma, B.; Adhikari, D.; Tanti, B. Habitat distribution modelling for improving conservation status of Brucea mollis Wall. ex Kurz.-An endangered potential medicinal plant of Northeast India. J. Nat. Conserv. 2018, 43, 104-110. [CrossRef]

17. Singh, P.B.; Mainali, K.; Jiang, Z.; Thapa, A.; Subedi, N.; Awan, M.N.; Ilyas, O.; Luitel, H.; Zhou, Z.; Hu, H. Projected distribution and climate refugia of endangered Kashmir musk deer Moschus cupreus in greater Himalaya, South Asia. Sci. Rep. 2020, 10, 1511. [CrossRef] [PubMed]

18. Cheng, W.C. Plantae novae Chekiangenses. In Contributions from the Biological Laboratory of the Science Society of China; Botanical Series; The Biological laboratory of the Science Society of China: Nanjing, China, 1932; Volume 8, pp. 135-142.

19. Li, P.C.; Skvortsov, A.K. Betulaceae. In Flora of China; Wu, C.Y., Raven, P.H., Eds.; Science Press: Beijing, China, 1999; Volume 4, pp. 289-300.

20. Shaw, K.; Roy, S.; Wilson, B. Carpinus tientaiensis. The IUCN Red List of Threatened Species 2014, e.T194617A2353175. Available online: https://www.iucnredlist.org/species/194617/2353175 (accessed on 1 August 2014). [CrossRef]

21. Chen, M.S.; Jin, Z.X.; Ke, S.X. Measurement and analysis of leaf shape variation of Carpinus tientaiensis in different light environment. Sci. Silvae Sin. 2018, 54, 54-63. [CrossRef]

22. Fick, S.E.; Hijmans, R.J. WorldClim 2: New 1-km spatial resolution climate surfaces for global land areas. Int. J. Climatol. 2017, 37, 4302-4315. [CrossRef]

23. Taylor, K.E.; Ronald, S.; Meehl, G.A. An overview of CMIP5 and the Experiment Design. Bull. Am. Meteorol. Soc. 2011, 93, 485-498. [CrossRef]

24. Phillips, S.J. Transferability, sample selection bias and background data in presence-only modelling: A response to Peterson et al. (2007). Ecography 2008, 31, 272-278. [CrossRef]

25. Elith, J.; Phillips, S.J.; Hastie, T.; Dudík, D.; Chee, Y.E.; Yates, C.J. A statistical explanation of MaxEnt for ecologists. Divers. Distrib. 2010, 17, 43-57. [CrossRef]

26. Raes, N.; Steege, H.T. A null-model for significance testing of presence-only species distribution models. Ecography 2007, 30, 727-736. [CrossRef] 
27. Fielding, A.H. What are the appropriate characteristics of an accuracy measure? In Predicting Species Occurrences: Issues of Accuracy and Scale; Scott, J.M., Heglund, P., Morrison, M.L., Raven, P.H., Eds.; Island Press: Washington, DC, USA, 2002; pp. 271-280.

28. Reineking, B.; Der, B.S. Constrain to perform: Regularization of habitat models. Ecol. Model. 2006, 193, 675-690. [CrossRef]

29. Khadka, K.K.; Kannan, R.; Ilyas, O.; Abbas, F.; James, D.A. Where are they? Where will they be? In pursuit of current and future whereabouts of endangered Himalayan musk deer. Mamm. Biol. 2017, 85, 30-36. [CrossRef]

30. Zhang, K.L.; Yao, L.J.; Meng, J.S.; Tao, J. Maxent modeling for predicting the potential geographical distribution of two peony species under climate change. Sci. Total Environ. 2018, 634, 1326-1334. [CrossRef]

31. Narouei-Khandan, H.A. Ensemble Models to Assess the Risk of Exotic Plant Pathogens in a Changing Climate. Master's Thesis, Lincoln University, Oakland, CA, USA, 2014.

32. Robertson, M.P.; Peter, C.I.; Villet, M.H.; Ripley, B.S. Comparing models for predicting species' potential distributions: A case study using correlative and mechanistic predictive modelling techniques. Ecol. Model. 2003, 164, 153-167. [CrossRef]

33. Williams, J.N.; Seo, C.; Thorne, J.; Nelson, J.K.; Erwin, S.; O’Brien, J.M.; Schwartz, M.W. Using species distribution models to predict new occurrences for rare plants. Divers. Distrib. 2009, 15, 565-576. [CrossRef]

34. Wisz, M.S.; Hijmans, R.J.; Li, J.; Peterson, A.T.; Graham, C.H.; Guisan, A. Effects of sample size on the performance of species distribution models. Divers. Distrib. 2008, 14, 763-773. [CrossRef]

35. Guisan, A.; Broennimann, O.; Engler, R.; Vust, M.; Yoccoz, N.G.; Lehmann, A.; Zimmermann, N.E. Using Niche-based models to improve the sampling of rare species. Conserv. Biol. 2006, 20, 501-511. [CrossRef]

36. Garcia, K.; Lasco, R.; Ines, A.; Lyon, B.; Pulhin, F. Predicting geographic distribution and habitat suitability due to climate change of selected threatened forest tree species in the Philippines. Appl. Geogr. 2013, 44, 12-22. [CrossRef]

37. Elith, J.; Graham, C.H.; Anderson, R.P.; Dudik, M.; Ferrier, S.; Guisan, A.; Hijmans, R.J.; Huettmann, F.; Leathwick, J.R.; Lehmann, A. Novel methods improve prediction of species' distributions from occurrence data. Ecography 2006, 29, 129-151. [CrossRef]

38. Hernández, P.A.; Graham, C.H.; Master, M.M.; Albert, D. The effect of sample size and species characteristics on performance of different species distribution modeling methods. Ecography 2006, 29, 773-785. [CrossRef]

39. Thomas, C.D.; Cameron, A.; Green, R.E.; Bakkenes, M.; Beaumont, L.J.; Collingham, Y.C.; Erasmus, B.F.N.; Siqueira, M.F.; Grainger, A.; Hannah, L.; et al. Extinction risk from climate change. Nature 2004, 427, 145-148. [CrossRef]

40. Iverson, L.R.; Prasad, A.M.; Matthews, S.N.; Peters, M.P. Estimating potential habitat for 134 eastern US tree species under six climate scenarios. Forest Ecol. Manag. 2008, 254, 390-406. [CrossRef]

41. Shaw, K.; Roy, S.; Wilson, B. Carpinus putoensis. The IUCN Red List of Threatened Species 2014, e.T32303A2813038. Available online: https://www.iucnredlist.org/species/32303/2813038 (accessed on 1 August 2014). [CrossRef]

42. Roy, S.; Shaw, K.; Wilson, B. Carpinus hebestroma. The IUCN Red List of Threatened Species 2016, e.T194577A2350438. Available online: https://www.iucnredlist.org/species/194577/2350438 (accessed on 1 August 2014). [CrossRef]

43. Lu, Z.Q.; Liu, J.Q.; Beech, E. Carpinus langaoensis. The IUCN Red List of Threatened Species 2018. Available online: https://www.iucnredlist.org/species/117839549/117839554 (accessed on 8 August 2017). [CrossRef]

44. Engler, R.; Guisan, A.; Rechsteiner, L. An improved approach for predicting the distribution of rare and endangered species from occurrence and pseudo-absence data. J. Appl. Ecol. 2004, 41, 263-274. [CrossRef]

45. Cai, R.S.; Tan, H.J.; Kontoyiannis, H. Robust surface warming in offshore china seas and its relationship to the East Asian monsoon wind field and ocean forcing on interdecadal time scales. J. Clim. 2017, 30, 8987-9005. [CrossRef]

46. Parmesan, C.; Yohe, G. A globally coherent fingerprint of climate change impacts across natural systems. Nature 2003, 421, 37-42. [CrossRef]

47. Li, S.S.; Lu, J.Y.; Yan, J.P.; Liu, X.; Kong, F.; Wang, J. Spatiotemporal variability of temperature in northern and southern Qinling Mountains and its influence on climatic boundary. Acta Geogr. Sin. 2018, 73, 13-24. [CrossRef] 
48. Burbano-Girón, J. Modeling the Dispersion of Atelines (Primates, Atelinae) Through Scenarios of Climate Change and Habitat Fragmentation in Colombia. Conservation Implications for the Persistence of Species into the Future. Master's Thesis, Universidad Nacional de Colombia, Bogotá, Colombia, 2013.

49. Hu, X.S.; Wu, C.Z.; Hong, W.; Qiu, R.Z.; Li, J.; Hong, T. Forest cover change and its drivers in the upstream area of the Minjiang River, China. Ecol. Indic. 2014, 46, 121-128. [CrossRef]

50. Yi, Y.J.; Zhou, Y.; Cai, Y.P.; Yang, W.; Li, Z.W.; Zhao, X. The influence of climate change on an endangered riparian plant species: The root of riparian Homonoia. Ecol. Indic. 2018, 92, 40-50. [CrossRef]

51. Yi, Y.J.; Tang, C.H.; Yang, Z.F.; Chen, X. Influence of Manwan Reservoir on fish habitat in the middle reach of the Lancang River. Ecol. Eng. 2014, 69, 106-117. [CrossRef]

52. Cunha, D.D.A.; Ferreira, L.V. Impacts of the Belo Monte hydroelectric dam construction on pioneer vegetation formations along the Xingu River, Pará State, Brazil. Braz. J. Bot. 2012, 35, 159-167. [CrossRef]

53. Zhu, J.Y.; Zhang, L.F.; Shen, P.; Ren, B.Q.; Liang, Y.; Chen, Z.D. Wind Pollination Characteristics of Styles in Betulaceae. Chin. Bull. Bot. 2014, 49,524-538. [CrossRef]

54. Vetaas, O.R. Realized and potential climate niches: A comparison of four Rhododendron tree species. J. Biogeogr. 2002, 29, 545-554. [CrossRef]

(C) 2020 by the authors. Licensee MDPI, Basel, Switzerland. This article is an open access article distributed under the terms and conditions of the Creative Commons Attribution (CC BY) license (http://creativecommons.org/licenses/by/4.0/). 